\title{
Host-Parasite Interaction in Patients with Infections due to Escherichia coli. I. The Serogrouping of E. coli from Intestinal and Extraintestinal Sources *
}

\author{
Kenneth L. Vosti, Leonard M. Goldberg, Arnold S. Monto, and \\ Lowell A. Rantz $\dagger$ \\ (From the Division of Infectious Disease, Department of Medicine, Stanford University \\ School of Medicine, Palo Alto, Calif.)
}

A serologic scheme for the classification of Escherichia coli was dependent upon the recognition by Kauffmann of heat stable group specific $O$ antigens (1). The presence of heat labile surface $\mathrm{K}$ and flagellar $\mathrm{H}$ antigens, which may be common to a number of $O$ groups, interfered with prior attempts to devise such a method. The clinical importance of this observation was emphasized initially by the recognition that certain strains of $E$. coli were commonly associated with diarrheal disease of the newborn (2). The relationship of the fecal flora to appendicitis has been explored; however, specific strains could not be implicated as a cause of this disease (3). Other investigations have reported studies of strains obtained from patients with infections of extraintestinal sites and have revealed that a few serogroups accounted for 60 to $85 \%$ of the groupable strains (4-11).

This communication presents an analysis of carefully defined human populations with extraintestinal infections due to $E$. coli. The information provided is relevant to the study of hostparasite interaction and is concerned with the following problems: 1) What is the reservoir for disease? 2) Does the reservoir differ among hosts with and without disease? 3) What is the influence of the portal of entry? 4) Are some $\mathrm{O}$ groups more likely to be associated with disease among different populations? 5) Do certain O groups occur more commonly with the more severe forms of the disease?

* Submitted for publication June 15, 1964 ; accepted August 27, 1964.

Supported by research grant no. A103638 and training grant no. TI AI 185 from the National Institute of Allergy and Infectious Diseases. Published in abstract form, J. clin. Invest. 1962, 41, 1408, and 1963, 42, 989.

† Deceased, June 1964.

\section{Methods}

Paticnts. Two hundred seventy-seven patients with infections of the urinary tract or bacteremia were studied from 1959 to 1963. In addition, fecal specimens were obtained from 102 prenatal women without evidence of infection of the urinary tract. All were observed during their hospitalization or attendance at the outpatient clinics of the Palo Alto-Stanford Hospitals.

Group I consisted of 78 women, who were referred to a special pyelonephritis study clinic by physicians from the community, with histories of recurrent disease, and who were observed for 1 month to 3 years. Their observed infections were neither acquired in the hospital nor related to pregnancy. Eighty-five per cent were between the ages of 19 and 39 . Intravenous pyelograms were performed on $75 \%$ of these patients; structural abnormalities were demonstrated on $15 \%$ of these. The serum creatinine or blood urea nitrogen was normal among $95 \%$ of 63 patients, and not done in the others. Seven patients had associated chronic diseases: three were diabetic, one was diabetic and had hypertension, one had hypertension, one developed multiple sclerosis during observation, and one had chronic phenacitin intoxication.

Group II comprised 116 women whose infections were recognized during their antepartum care in the obstetrical outpatient clinic or during their immediate postpartum period in the hospital. Asymptomatic patients were detected by a routine screening program, which included an initial quantitative culture of the urine of all obstetrical patients at their first visit and follow-up cultures in each trimester, in the first 5 days postpartum, and at 6 weeks postpartum. Patients with symptomatic disease were studied when they presented with disease. The majority of infections observed during the antepartum period were evident at the first visit; however, three patients each had a single recurrence with a different serogroup during the antepartum period. A normal culture during the third trimester or from the delivery room was a prerequisite for inclusion in the group of patients with postpartum infections. All ladies were between the ages of 15 and 39 ; most did not have an intravenous pyelogram or determination of serum creatinine or blood urea nitrogen, and only one had an associated chronic disease. 
Group III consisted of 60 hospitalized patients with bacteremia. Twenty-one per cent were less than 10 years of age, and $47 \%$ were 60 or older. Approximately $90 \%$ of these patients had a serious congenital or acquired underlying disease or compromised renal function, or both.

Group IV comprised 102 pregnant women who did not have infections of the urinary tract. Specimens of urine and feces were obtained at their first or second visit to the antepartum clinic.

Definitions. Episodes of bacteriuria were classified by the following criteria: 1) confirmed bacteriuria-two or more serial cultures containing 100,000 or more colonies of $E$. coli per $\mathrm{ml}$ of urine in a patient without symptoms; at least one culture with 10,000 or more colonies of E. coli per $\mathrm{ml}$ in a patient with symptoms (over $90 \%$ had counts greater than 100,000 ); or any number of $E$. coli in a urine obtained by suprapubic aspiration of the bladder; 2) asymptomatic bacteriuria-confirmed bacteriuria in a patient who was free of symptoms referable to the genitourinary system; 3) cystitis-confirmed bacteriuria in a patient who complained of dysuria, nocturia, urgency, postmicturition urgency or hematuria, who was usually afebrile, and who had no costovertebral angle pain; 4) acute pyclonephritis-confirmed bacteriuria in a patient who may have complained of many of the symptoms associated with cystitis, but who, in addition, had fever, shaking chills, back or costovertebral pain, and general malaise.

Clinical materials. Specimens of urine were obtained as mid-stream voided samples or by suprapublic needle aspirations of the bladder. Specimens of feces were obtained with a rectal swab, vaginal specimens by a swab of the mid-vaginal wall or cervical area, and blood by puncture of the antecubital or femoral sites.

Bacteriology. The clinical materials were inoculated on blood, eosinmethylene blue, and MacConkey agar plates. Culture of the blood was performed initially in brain heart infusion broth. Quantitative cultures of the urine were done by plating it $\mathrm{ml}$ of undiluted and of $10^{-2}$ and $10^{-4}$ dilutions of urine on the above differential media. The urine was spread over the surface of the media with an L-shaped glass dolly, and the plates were incubated overnight at $37^{\circ} \mathrm{C}$. The colonies per milliliter of urine were calculated from a plate containing a countable number.

Fifteen to 25 colonies of typical E. coli were selected from each stool, and two to five from each specimen obtained from an extraintestinal source. All strains of E. coli failed to utilize citrate, were methyl red and indol positive, and fermented lactose rapidly. Late lactose fermenting bacteria, which otherwise resembled $E$. coli, were included in the study.

Serologic classification. Unabsorbed $O$ antisera were prepared by immunization of rabbits with standard $O$ antigens of E. coli. 1 Sera for $131 \mathrm{O}$ groups were available. Recognized $O$ groups that were missing included

1 Obtained from the Communicable Disease Center, U. S. Public Health Service, Atlanta, Ga.
$37,66,68,87,88,92,95,107,129,130,131,134, \mathrm{X} 3, \mathrm{X} 5$, $\mathrm{X} 6$, and $\mathrm{X7}$.

The technique for $\mathrm{O}$ grouping was that reported by Edwards and Ewing (12) and may be briefly summarized. Each of the individual strains of $E$. coli was grown in tryptose broth for 6 to 8 hours at $37^{\circ} \mathrm{C}$ and heated at $100^{\circ} \mathrm{C}$ for 1 hour in an Arnold sterilizer. The final suspensions were used as the test antigens. The antisera were grouped into pools in which each member had a final dilution of $1: 100$. The tests were conducted by adding $0.1 \mathrm{ml}$ of pooled serum to $0.9 \mathrm{ml}$ of antigen suspension (final individual serum dilution. $1: 1,000)$. The mixtures were incubated at $50^{\circ} \mathrm{C}$ for 18 hours in a water bath and were read for visible agglutination by agitation of the tubes. Each strain was tested initially against all pools. Organisms that agglutinated in any pool or pools were retested against the individual sera in a similar manner. The individual sera were initially diluted $1: 50$; therefore, the final dilution in the test mixture was 1:500. Those strains reacting equally with more than one individual serum were again tested using serial twofold dilutions of each reactive serum. The highest titer determined the final assigned $\mathrm{O}$ group in the small number of strains that were examined in this manner. Absorbed sera were not employed to differentiate among cross-reacting strains in this study.

\section{Results}

Distribution of $O$ groups among specimens from various sources. E. coli were isolated and submitted for serogrouping from 573 specimens obtained from 356 patients. Simultaneous cultures of urine or stool, or both, and vaginal secretions were secured from 105 of those with infection; only urine from an additional 89 with infection; only blood from another 60 with bacteremia; and only feces from 102 patients without infection.

As shown in Table I, 800 different strains were identified among 4,651 colonies. Although 15 to 25 colonies were studied per stool specimen, each different serogroup is represented only once per individual specimen in Table I. Thus a specimen from group I containing 15 colonies of 06,5 of 04 , and 5 that were rough would appear as 1 opposite 06,04 , and rough in the column under group I. Extraintestinal specimens are charted similarly.

Seventy-nine per cent of the different strains were serologically classified; $14 \%$ were smooth but were not agglutinated by any of the antisera, and $7 \%$ were rough and therefore not suitable for O grouping. Sixty-nine different serogroups were identified among all the specimens: 66 among 
TABLE I

Distribution of serogroups of E. coli identified among various human materials

\begin{tabular}{|c|c|c|c|c|c|c|}
\hline O group & $\begin{array}{c}\text { Normal } \\
\text { stool }\end{array}$ & $\begin{array}{l}\text { Disease } \\
\text { stool }\end{array}$ & Vagina & Urine & Blood & Total \\
\hline 1 & 16 & 17 & 2 & 11 & 3 & 46 \\
\hline 2 & 0 & 1 & 1 & 2 & 0 & 4 \\
\hline 3 & 1 & 2 & 0 & 0 & 0 & 3 \\
\hline 4 & 6 & $1 \overline{8}$ & 11 & 33 & 11 & 79 \\
\hline 5 & 1 & 0 & 0 & 3 & 1 & 5 \\
\hline 6 & 11 & 32 & 22 & 39 & 15 & 119 \\
\hline 7 & 15 & 7 & 4 & 8 & 2 & 36 \\
\hline $8 / 93$ & 2 & 1 & 1 & 2 & 1 & 7 \\
\hline 9 & 3 & 2 & 0 & 0 & 0 & 5 \\
\hline 11 & 3 & 4 & 1 & 4 & 0 & 12 \\
\hline 12 & 3 & 1 & 0 & 1 & 1 & 6 \\
\hline 13 & 0 & 2 & 0 & 1 & 1 & 4 \\
\hline 15 & 3 & 1 & 0 & 3 & 2 & 9 \\
\hline $16 / 62$ & 4 & 3 & 3 & 12 & 1 & 23 \\
\hline $17 / 77$ & 1 & 2 & 2 & 4 & 2 & 11 \\
\hline 18 & 7 & 3 & 2 & 6 & 0 & 18 \\
\hline 19 & 1 & 2 & 0 & 1 & 0 & 4 \\
\hline 21 & 7 & 2 & 0 & 0 & 1 & 10 \\
\hline 22 & 0 & 1 & 2 & 4 & 0 & 7 \\
\hline 23 & 3 & 1 & 2 & 2 & 0 & 8 \\
\hline 25 & 5 & 8 & 3 & 9 & 2 & 27 \\
\hline 28 & 1 & 5 & 1 & 1 & 0 & 8 \\
\hline 36 & 1 & 2 & 0 & 0 & 0 & 3 \\
\hline 39 & 1 & 0 & 0 & 1 & 0 & 2 \\
\hline 40 & 3 & 4 & 0 & 0 & 0 & 7 \\
\hline 41 & 1 & 1 & 0 & 0 & 0 & 2 \\
\hline $48 / 59$ & 4 & 3 & 0 & 1 & 0 & 8 \\
\hline 49 & 0 & 1 & 0 & 1 & 0 & 2 \\
\hline 50 & 3 & 12 & 6 & 13 & 3 & 37 \\
\hline 51 & 1 & 0 & 0 & 1 & 0 & 2 \\
\hline 55 & 1 & 0 & 1 & 1 & 0 & 3 \\
\hline 75 & 8 & 9 & 4 & 10 & 3 & 34 \\
\hline 81 & 1 & 1 & 1 & 1 & 0 & 4 \\
\hline 83 & 0 & 3 & 1 & 2 & 0 & 6 \\
\hline 101 & 0 & 0 & 1 & 1 & 0 & 2 \\
\hline 111 & 0 & 0 & 0 & 0 & 1 & 1 \\
\hline 113 & 1 & 1 & 1 & 1 & 0 & 4 \\
\hline 118 & 0 & 0 & 0 & 1 & 0 & 1 \\
\hline 128 & 5 & 1 & 0 & 0 & 0 & 6 \\
\hline 132 & 0 & 1 & 1 & 1 & 0 & 3 \\
\hline $\mathrm{OX} 2$ & 1 & 2 & 1 & 0 & 0 & 4 \\
\hline $\mathrm{A}^{*}$ & 23 & 0 & 0 & 0 & 0 & 23 \\
\hline $\mathrm{B} \dagger$ & 0 & 17 & 0 & 0 & 0 & 17 \\
\hline No. different & & & & & & \\
\hline $\mathrm{O}$ groups & 49 & 45 & 22 & 32 & 16 & 69 \\
\hline No. groupable & & & & & & \\
\hline strains & 148 & 174 & 74 & 181 & 50 & 627 \\
\hline $\begin{array}{l}\text { No. nongroup- } \\
\text { able strains }\end{array}$ & 43 & 27 & 12 & 31 & 5 & 118 \\
\hline No. rough & & & & & & \\
\hline strains & 16 & 16 & 4 & 14 & 5 & 55 \\
\hline Total of differ- & & & & & & \\
\hline ent strains & 207 & 217 & 90 & 226 & 60 & 800 \\
\hline No. of colonies & 1,503 & 1,969 & 375 & 704 & 120 & 4,651 \\
\hline $\begin{array}{l}\text { No. of speci- } \\
\text { mens }\end{array}$ & 102 & 117 & 79 & 215 & 60 & 573 \\
\hline $\begin{array}{l}\text { No. of pa- } \\
\text { tients }\end{array}$ & 102 & 105 & 72 & 194 & 60 & 356 \\
\hline
\end{tabular}

*A: one strain of groups $30,59,74,78,84,85,91,93,103,108,114,119$, and OX4; two strains of groups 86 and 117 ; three strains of groups 133 and 135 .

$\dagger \mathrm{B}$ : one strain of groups $52,82,98,102,104,110,142$, and $\mathrm{OX} 8$; two strains of group 20 ; three strains of group 27 ; and four strains of group 10. 
feces, 32 among urine, 22 among vaginal secretions, and 16 among blood. The number of groupable strains from each of the latter two sources was less than one-half of those studied from either feces or urine. Thirty-six $O$ groups were identified in feces that were not found in urine; single strains of only two serogroups (0101, 0118) were found in urine that were not recognized in feces.

Eight $(1,4,6,7,16 / 62,25,50$, and 75$)$ of the 69 serogroups accounted for $63 \%$ of the total number of groupable strains and ranged from $46 \%$ among specimens of normal feces to $80 \%$ among those of blood. Four and 6 were the most frequently isolated serogroups among specimens from each of the different sources. The frequency rank of the other six serogroups varied with the source of the material.

Sixteen strains were identified that might represent enteropathogenic E. coli, $3 \%$ of the total number of groupable strains. Ten, or $3 \%$ of the groupable strains from this source, were found in feces.

Comparison of $O$ groups among specimens of urine from different populations. Table II presents a comparison of the common serogroups identified in specimens of urine obtained from two distinct study populations. Patients in group I were not pregnant and acquired their infections

TABLE II

Occurrence of the common serogroups among specimens of urine

\begin{tabular}{lrrr}
\hline & & \multicolumn{2}{c}{ Group II } \\
\cline { 3 - 4 } Common O groups & & $\begin{array}{c}\text { Ante- } \\
\text { partum }\end{array}$ & $\begin{array}{c}\text { Post- } \\
\text { partum }\end{array}$ \\
\hline 1 & Group I* & 3 & 6 \\
4 & 18 & 4 & 11 \\
6 & 23 & 5 & 11 \\
7 & 2 & 3 & 3 \\
$16 / 62$ & 3 & 1 & 8 \\
25 & 7 & 0 & 2 \\
50 & 7 & 1 & 5 \\
75 & 5 & 3 & 2 \\
No. different O groups & 18 & 13 & 23 \\
Total common O groups & 67 & 20 & 48 \\
Total other O groups & 15 & 8 & 23 \\
No. nongroupable strains & 14 & 3 & 14 \\
No. rough strains & 4 & 3 & 7 \\
Total different strains & 100 & 34 & 92 \\
No. specimens & 94 & 33 & 88 \\
No. patients & 78 & 29 & 87 \\
& & &
\end{tabular}

* Study clinic.

† Obstetric.
TABLE III

Occurrence of the common serogroups among feces obtained from patients with and without infection of the urinary tract

\begin{tabular}{lrrr}
\hline \multicolumn{1}{c}{ Common O group } & Group I* & Group II $\dagger$ & Group IV $\ddagger$ \\
\hline 1 & 8 & 6 & 16 \\
6 & 9 & 5 & 6 \\
7 & 17 & 11 & 11 \\
$16 / 62$ & 5 & 1 & 15 \\
25 & 1 & 2 & 4 \\
50 & 4 & 3 & 5 \\
75 & 4 & 6 & 3 \\
No. different O groups & 2 & 6 & 8 \\
Total common O groups & 30 & 29 & 49 \\
Total other O groups & 50 & 40 & 68 \\
No. nongroupable strains & 29 & 31 & 80 \\
No. rough strains & 15 & 9 & 43 \\
Total different strains & 6 & 5 & 16 \\
No. of specimens & 100 & 85 & 207 \\
No. of patients & 53 & 41 & 102 \\
No. of colonies & 47 & 39 & 102 \\
Total no. 4, 6, 50 & 959 & 680 & 1,503 \\
None of common O groups & 30 & 22 & 20 \\
& 14 & 10 & 46 \\
\hline
\end{tabular}

* Study clinic, infected.

Obstetric, postpartum, infected.

Obstetric, antepartum, not infected.

during residence in the community; those in group II were pregnant and acquired their infections before or during their antepartum visits, or during hospitalization for delivery and immediate postpartum care.

Serologic identification was possible in $82 \%$ of the strains from group I and in $82 \%$ of antepartum and $77 \%$ of postpartum strains from group II. Eighteen different $\mathrm{O}$ groups were found among the former, and 13 and 23, respectively, among the latter. The eight common $\mathrm{O}$ groups accounted for $82 \%$ of the strains that were identified serologically from group I, and for $71 \%$ of antepartum and $68 \%$ of postpartum strains from group II. Serogroups 1, 7, and 16/62 were identified more frequently among specimens from group II than I; 04, 6, and 25 occurred more commonly among those from group I. Serogroups 50 and 75 were equally distributed.

Comparison of common $O$ groups among specimens of feces from different populations. Table III illustrates the distribution of the common serogroups among specimens of feces from groups I (study clinic) and II (obstetrical, postpartum). The distribution in these populations with infections of the urinary tract was contrasted with that among patients in group IV who attended the antepartum clinic and did not have infections. 
The numbers present in each category were derived as defined for Table I.

Neither the frequency of occurrence of individual $O$ groups nor the number of specimens containing none of the common $\mathrm{O}$ groups varied among specimens from groups I and II. However, nearly twice as many of the specimens from patients without disease failed to contain any of the common O groups. Serogroups 4, 6, and 50 were isolated 3 times more frequently from the feces of patients with disease than among those of normal patients. Serogroups 1, 16/62, and 75 were distributed evenly, whereas 07 was isolated more frequently from normal feces.

The number of specimens containing more than one or two serogroups was not different among specimens obtained from patients with and without disease. A few specimens contained as many as six different serogroups; however, approximately $75 \%$ of those in either group contained only one or two.

Comparison of serogroups identified among paired specimens. The same strains were found in $80 \%$ of 97 paired specimens of urine and feces obtained simultaneously from infected patients before the initiation of treatment. This value did not differ appreciably among specimens obtained from patients in groups I and II. The urinary pathogen represented the only or predominant strain isolated from nearly $75 \%$ of the

TABLE IV

Relationship of serogroups to the various clinical syndromes

\begin{tabular}{|c|c|c|c|c|c|c|c|c|c|c|}
\hline \multirow[b]{2}{*}{ O group } & \multicolumn{3}{|c|}{$\begin{array}{c}\text { Asymptomatic } \\
\text { bacteriuria }\end{array}$} & \multicolumn{3}{|c|}{ Cystitis } & \multicolumn{3}{|c|}{$\begin{array}{l}\text { Acute pyelo- } \\
\text { nephritis }\end{array}$} & \multirow{2}{*}{$\begin{array}{l}\text { Racte- } \\
\text { remiaf }\end{array}$} \\
\hline & $I^{*}$ & IIt & Total & I & II & Total & I & II & Total & \\
\hline $\begin{array}{l}1 \\
2\end{array}$ & & 2 & 2 & 1 & $\begin{array}{l}5 \\
2\end{array}$ & $\begin{array}{l}6 \\
2\end{array}$ & & 2 & 2 & \\
\hline 4 & & 5 & 5 & 12 & 8 & 20 & 6 & 2 & 8 & 9 \\
\hline 5 & 1 & & 1 & & & & & 1 & 1 & 1 \\
\hline 6 & 2 & 11 & 13 & 15 & 4 & 19 & 8 & 1 & 9 & 10 \\
\hline 7 & & 1 & 1 & 2 & & 2 & & 5 & 5 & 1 \\
\hline $8 / 93$ & 1 & & 1 & 1 & & 1 & & & & 1 \\
\hline 11 & & 2 & 2 & & 2 & 2 & & & & \\
\hline 12 & & & & & 1 & 1 & & & & \\
\hline 13 & & & & & 1 & 1 & & & & \\
\hline 15 & 1 & & 1 & 1 & & 1 & & & & 2 \\
\hline $16 / 62$ & & 2 & 2 & 1 & 1 & 2 & 1 & 6 & 7 & 1 \\
\hline $17 / 77$ & & 2 & 2 & 2 & & 2 & & & & 1 \\
\hline 18 & & 1 & 1 & 1 & 2 & 3 & & 1 & 1 & \\
\hline $19^{\circ}$ & & & & & 1 & 1 & & & & \\
\hline 22 & & 1 & 1 & 2 & 1 & 3 & & & & \\
\hline 23 & & 1 & 1 & & 1 & 1 & & & & \\
\hline 25 & 2 & & 2 & 5 & 2 & 7 & & & & 2 \\
\hline 28 & & & & & & & & 1 & 1 & \\
\hline 39 & & & & 1 & & 1 & & & & \\
\hline 49 & & & & & & & & 1 & 1 & \\
\hline 50 & & 1 & 1 & 3 & 3 & 6 & 5 & 2 & 7 & 2 \\
\hline 55 & & & & & 1 & 1 & & & & \\
\hline 75 & 2 & 1 & 3 & 3 & 3 & 6 & & 1 & 1 & 2 \\
\hline 81 & & & & & 1 & 1 & & & & \\
\hline 83 & & 1 & 1 & & 1 & 1 & & & & \\
\hline 101 & & & & 1 & & 1 & & & & \\
\hline 113 & & 1 & 1 & & & & & & & \\
\hline 118 & & 1 & 1 & & & & & & & \\
\hline 132 & & 1 & 1 & & & & & & & \\
\hline No. different $\mathrm{O}$ groups & 6 & 16 & 20 & 15 & 18 & 24 & 4 & 11 & 11 & 11 \\
\hline No. groupable strains & 9 & 34 & 43 & 51 & 40 & 91 & 20 & 23 & 43 & 32 \\
\hline No. nongroupable strains & 4 & 7 & 11 & 6 & 7 & 13 & 3 & 3 & 6 & 2 \\
\hline No. rough strains & 1 & 5 & 6 & 2 & 2 & 4 & 0 & 3 & 3 & 3 \\
\hline Total different strains & 14 & 46 & 60 & 59 & 49 & 108 & 23 & 29 & 52 & 37 \\
\hline No. of specimens & 13 & 46 & 59 & 57 & 45 & 102 & 22 & 28 & 50 & 37 \\
\hline No. of patients & 12 & 46 & 58 & 48 & 45 & 93 & 22 & 28 & 50 & 37 \\
\hline
\end{tabular}

* Study clinic.

† Obstetric.

$\ddagger$ Secondary to urinary tract portal of entry. 
cultures of feces. The same strains were identified among $92 \%$ of 66 paired urine and vaginal specimens.

Multiple cultures were available from 19 patients with bacteremia secondary to a portal of entry in the urinary tract. The same strains were identified in paired specimens of blood and urine from 18 of 19 patients, and in paired stool and blood specimens from eight of nine. In seven of eight patients, the same strains were present in blood, urine, and stool specimens.

Comparison of $O$ group with clinical syndrome. The distribution of serogroups of $E$. coli identified in specimens of urine and blood obtained from patients with different clinical syndromes and the influence of different study populations on this distribution are presented in Table IV. The proportion of strains classified varied from $72 \%$ among patients with asymptomatic bacteriuria to $87 \%$ among those with bacteremia; the proportion of those that could not be classified or were rough varied little among specimens from patients with different clinical syndromes.

The greatest number of different $O$ groups was found among specimens obtained from patients with cystitis and asymptomatic bacteriuria, the fewest among those from patients with acute pyelonephritis. Four different serogroups were identified in group I, as compared to 11 in group II.

The relationship of eight common serogroups to the different clinical syndromes is shown in Figure 1. The common $O$ groups comprised $68 \%$ of the total number of groupable strains isolated among specimens obtained from patients with asymptomatic bacteriuria: $75 \%$ with cystitis, $91 \%$ with acute pyelonephritis, and $84 \%$ with bacteremia. Four and 6 were the most frequently identified $O$ groups among each of the clinical syndromes and accounted for approximately the same percentage of the total number of strains that was classified among each. Of the common serogroups only $7,16 / 62$, and 50 were identified more frequently among strains isolated from patients with acute pyelonephritis than among those from patients with less severe clinical syndromes. Their occurrence in patients with bacteremia, however, was similar to that in patients with asymptomatic bacteriuria and cystitis.

The distribution of common $\mathrm{O}$ groups among specimens of urine obtained from patients with cystitis did not vary in the two study populations.

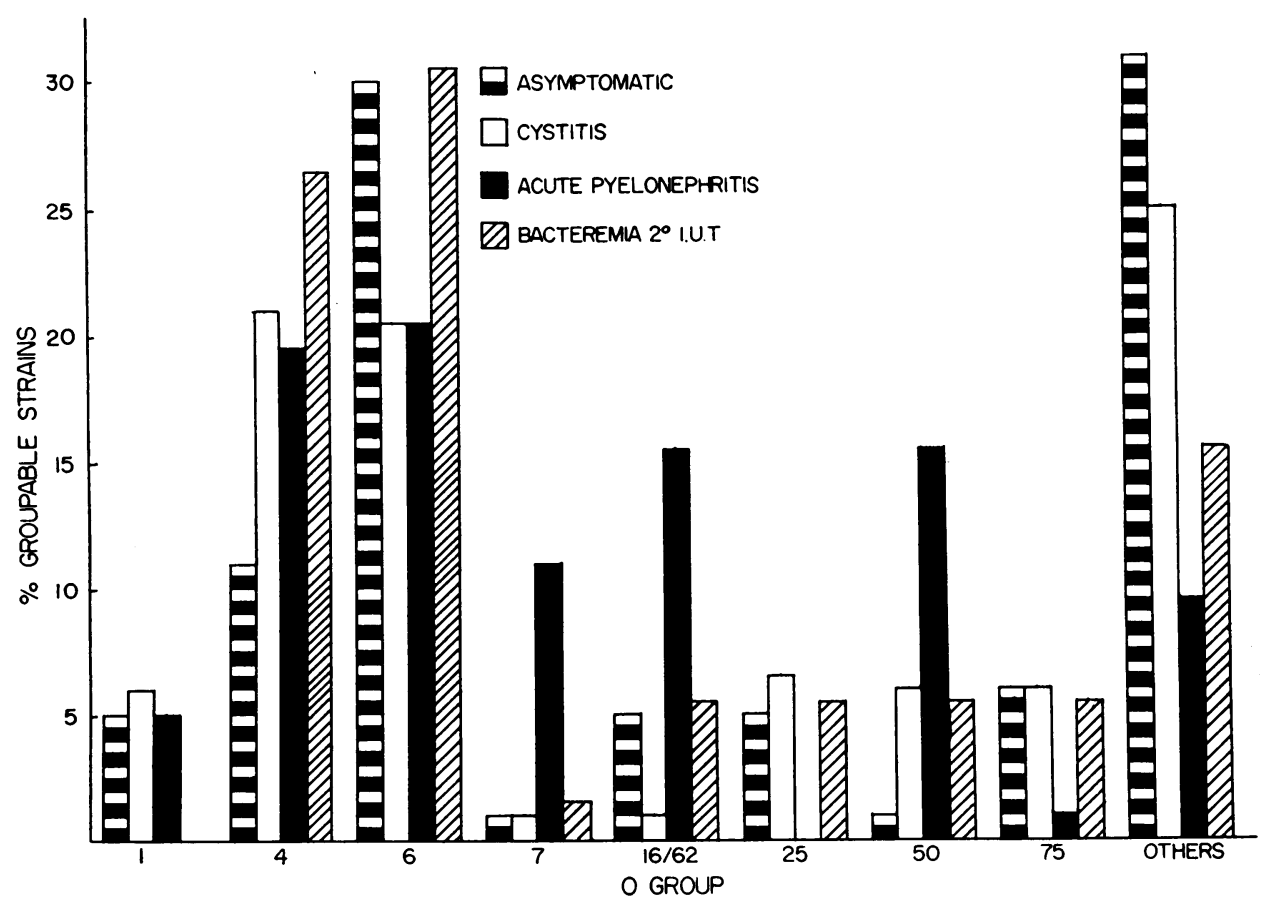

Fig. 1. OCCURRence of the COMMON SERogroups among the various Clinical syndromes. 


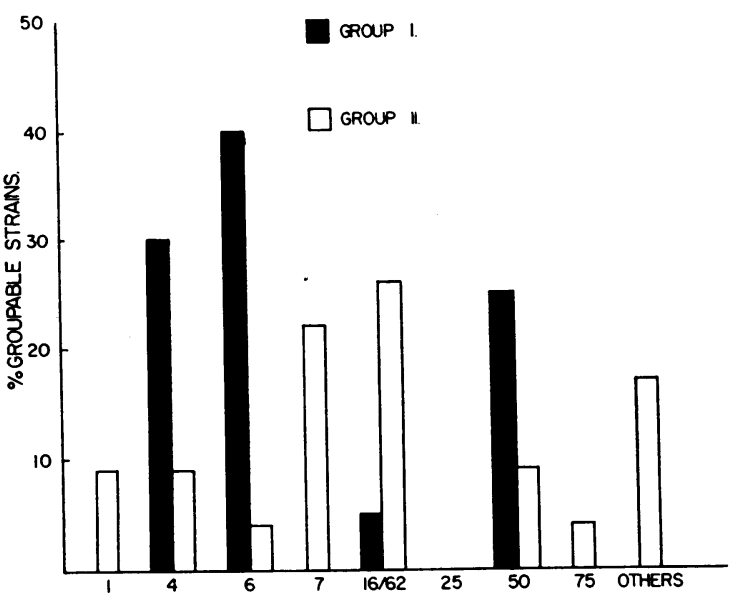

Fig. 2. CoMparison OF the COMMON SEROGROUPS AMONG PATIENTS WITH ACUTE PYELONEPHRITIS FROM TWO DIFFERENT POPLLATIONS.

A comparison among those with asymptomatic bacteriuria was not possible as group I was too small. A study of the two populations with acute pyelonephritis, however, revealed marked differences. Figure 2 shows that serogroups 4 and 6 were identified most frequently among specimens from patients in group I and 7 and 16/62 among those from group II. Serogroups 4, 6, and 50 accounted for $95 \%$ of the groupable strains among patients from group I and only $22 \%$ of those among patients from group II. In contrast, 01, $7,16 / 62$, and 75 accounted for $61 \%$ of the groupable strains among specimens from group II and only $5 \%$ of those among specimens from group I.

\section{Discussion}

The multiplicity of factors that may influence host-parasite interactions makes an accurate assessment of such relationships difficult even under the most rigidly controlled laboratory conditions. An appraisal of this interaction in man is even more tenuous when the many hereditary and acquired extra- and intraenvironmental changes that may profoundly alter host susceptibility or response to a given parasite are considered. Additionally, no guarantee exists that similar pathogens, as judged by bacteriologic and serologic methods of classification, isolated from different humans possess the same biologic potential to cause disease. Such reservations, however, should only qualify, but not discourage attempts, such as the present study, to gain a better understanding of host-parasite interactions in man.

What was the reservoir for infection? The reservoir for human infections with $E$. coli has generally been assumed to be the patient's own fecal flora. The difficulties in assessing the number of different serogroups in feces and the constant turnover in fecal strains of $E$. coli have been well documented (13-15).

Ideally, one would have liked to obtain a stool specimen just before the development of infection in the urinary tract to eliminate the possibility of retrograde colonization of the feces with the infecting strain from the urine. However, this was not possible, and therefore specimens obtained at the time of infection have been utilized for this purpose. Our studies have revealed that in $80 \%$ of simultaneously collected specimens of urine and feces the infecting strain from the urine could be identified in the feces. Although the above objection cannot be excluded, it is the authors' belief that these data support the contention that the patient's own feces was the immediate reservoir for infection. The remaining $20 \%$ may have represented true exogenous introduction of organisms, but probably reflected the limitations of the sampling techniques or the constant turnover in the coliform flora of feces. Similar results have been reported by Turck and Petersdorf (10).

Did the fecal reservoir differ among patients with and without disease? Of the serogroups that were commonly associated with infections of the urinary tract, 04,6 , and 50 were isolated 3 times more frequently from the feces of patients with infection than among those without; 01, $16 / 62$, and 75 were distributed evenly, and 07 was isolated more frequently among those without infection. Approximately twice as many of the fecal specimens from patients without infections failed to contain one of these $O$ groups as compared to those from patients with infections. Their distribution among patients whose infections were acquired in the hospital or in the community was similar. These observations are at variance with those reported by Turck and Petersdorf (10) and may be related to their use of feces from hospitalized patients as "normal" controls.

What was the influence of the portal of entry? The distribution of eight common $\mathrm{O}$ groups (1, 
$4,6,7,16 / 62,25,50$, and 75$)$ was not influenced by the portal of entry. They comprised 68 to $84 \%$ of the groupable strains among specimens obtained from various extraintestinal sources. This range was in general agreement with the reports of others (4-11) and was compatible with wide geographic associaton of these common eight groups with extraintestinal infections in both this country and Europe.

Were some $\mathrm{O}$ groups more likely to be associated with disease among different populations? Patients with infections of the urinary tract have been the most frequently studied group with extraintestinal disease. The eight common serogroups accounted for $75 \%$ of the groupable strains isolated from specimens obtained from patients with confirmed infections of the urinary tract in this study. These results were in general agreement with early reports (4-6), but 04 and 6 have occurred more frequently in this and other recent studies (7-11).

The total distribution of the common $O$ groups did not differ between two different study populations with infections of the urinary tract, although that of the individual $O$ groups varied. Serogroups 4, 6, 25, 50, and 75 were isolated more frequently from nonpregnant outpatients (group I) than among ante- or postpartum patients (group II), whereas 01,7 , and $16 / 62$ were more common among the latter. Only 016/62 occurred with a greater frequency among hospitalized postpartum than among antepartum patients and therefore might suggest a nosocomial relationship. A comparison of the distribution of O groups in the populations reported in the present studies with that reported by others was not possible, since most authors failed to record a clinical characterization of their patients with respect to age, associated normal or abnormal physiological states, and functional or structural renal abnormalities. These results do not differ significantly from those reported by Turck and Petersdorf (10) among an older population with a greater incidence of structural and functional abnormalities or from those of Kunin and Halmagyi (9) among children with asymptomatic bacteriuria.

Did certain O groups occur more commonly with the more severe forms of the disease? The virulence of a particular microorganism was de- fined by Miles (16) as the degree of pathogenicity of a strain in a particular host under defined conditions and can be expressed only in comparative terms because of variations in both host and parasite. The present study attempted to derive such information from an analysis of patients with clearly defined clinical syndromes and without obvious structural abnormalities of the urinary tract. The eight common $\mathrm{O}$ groups accounted for 68 to $91 \%$ of the groupable strains among patients with asymptomatic bacteriuria, cystitis, and acute pyelonephritis. Serogroups 4 and 6 were the most frequently identified in each syndrome, and they occurred with approximately the same frequency in each. Serogroups 7, 16/62, and 50 appeared to be disproportionately associated with acute pyelonephritis and hence to be more virulent. The hazard of attempting such an extrapolation was clear when the different populations were examined. Serogroups 4,6 , and 50 accounted for disease in $95 \%$ of the patients with acute pyelonephritis in group I and in only $22 \%$ of those in group II, whereas 1, 7, 16/62, and 75 accounted for $61 \%$ of those in group II and only $5 \%$ of those in group I. Thus, entirely different conclusions would have been drawn had only one or the other population been studied. It was probable that none of the common $\mathrm{O}$ groups differed with respect to virulence and that the observed results reflect population differences. The total distribution of common serogroups among these clinical syndromes was compared with that among patients with bacteremia secondary to a urinary portal of entry. The latter population was more heterogeneous in age and associated complications. None of the common $\mathrm{O}$ groups was more likely to occur with bacteremia than with the other clinical syndromes.

If virulence is defined as the ability to be associated with the more severe clinical forms of disease rather than the less, these eight common serogroups are not more virulent. If virulence is defined as the ability to survive in the environment and to be more commonly associated with disease, however, these strains are more virulent.

\section{Summary}

The ability to serogroup Escherichia coli prompted this study of the host-parasite interaction in patients with extraintestinal infections. 
Over 3,000 colonies were isolated from 471 specimens obtained from 254 patients with infections of the urinary tract or bacteremia, or both.

Serogroups $1,4,6,7,16 / 62,25,50$, and 75 accounted for approximately $80 \%$ of the groupable strains isolated from specimens of blood or urine. Serogroups 4 and 6 were identified most frequently and comprised $40 \%$ of those from urine and $52 \%$ of those from blood. The total proportion of the eight common $\mathrm{O}$ groups did not vary among specimens obtained from different human populations: however, the individual serogroups showed minor variations.

A study of the serogroups of $E$. coli in paired cultures of urine and feces revealed the same strain in both specimens of $80 \%$ of those examined. Fecal specimens obtained from infected patients were 3 to 4 times as likely to contain $\mathrm{O}$ groups 4.6 . or 50 as were those from healthy outpatients.

The distribution of the eight common serogroups among specimens obtained from patients with symptomatic bacteriuria, cystitis, acute pyelonephritis, and bacteremia secondary to a urinary tract portal of entry was compared. The disproportionate association of $\mathrm{O}$ groups $7,16 / 62$, and 50 with acute pyelonephritis appeared to reflect the population studied rather than enhanced virulence.

\section{Acknowledgments}

We greatly appreciated the technical skills and assistance of Miss Elizabeth Randall and the Mesdames $P$. Austill, F. Graham, M. Kocker, R. Lockman, and S. Schneider and the secretarial assistance of Mrs. H. Rantz and Mrs. J. Steiner.

\section{References}

1. Kauffmann, F. The serology of the coli group. J. Immunol. 1947, 57, 71.

2. Ewing, W. H. Enteropathogenic Escherichia coli serotypes. Ann. N. Y. Acad. Sci. 1956, 66, 61.

3. Vahlne, G. Serological typing of the colon bacteria, with special reference to the occurrence of $E$. coli in man under normal and pathological conditions, particularly in appendicitis. Acta path. microbiol. scand. (suppl.) 1945, 62, 1.

4. Grönroos, J. A., E. Mustakallio, and S. Virtanen. Serological identification of Escherichia coli strains from urinary infections and suppurative conditions. Ann. Med. exp. Fenn. 1955, 33, 133.

5. Leppanen, M. K. Investigations of Escherichia coli in acute appendicitis and in normal appendices and faeces with special reference to $O$ groups 1-25. Ann. Med. exp. Fenn. (suppl. 2) 1958, 36, 1.

6. Ujváry, G. I. Über die ätiologische Rolle der Escherichia-coli-Gruppee bei extraenteral lokalisierten Infektionen: Biochemische und serologische Untersuchungen. Zbl. Bakt., I. Abt. Orig. 1958, 170, 394.

7. Ewing, W. H., and B. R. Reavis. The O Antigen Groups of Escherichia coli Cultures from Various Sources. Atlanta, Ga., Communicable Disease Center, 1961.

8. Rantz, L. A. Serological grouping of Escherichia coli. Study in urinary tract infection. Arch. intern. Med. 1962, 109, 37.

9. Kunin, C. M., and N. E. Halmagyi. Urinary-tract infections in schoolchildren. II. Characterization of invading organisms. New Engl. J. Med. 1962, 266, 1297.

10. Turck, M., and R. G. Petersdorf. The epidemiology of non-enteric Escherichia coli infections: prevalence of serological groups. J. clin. Invest. 1962, 41, 1760.

11. Jackson, G. G., J. A. Arana-Sialer, B. R. Andersen, H. C. Grieble, and W. R. McCabe. Profiles of pyelonephritis. Arch. intern. Med. 1962, 110, 663.

12. Edwards, P. R., and W. H. Ewing. Identification of Enterobacteriaceae. Minneapolis, Burgess, 1955.

13. Vosti, K. L., A. S. Monto, and L. A. Rantz. The importance of sample size in studies based upon the serologic classification of Escherichia coli. Proc. Soc. exp. Biol. (N. Y.) 1962, 111, 201.

14. Sears, H. J., I. Brownlee, and J. K. Uchiyama. Persistence of individual strains of Escherichia coli in the intestinal tract of man. J. Bact. 1950, 59, 293.

15. Sears, H. J., and I. Brownlee. Further observations on the persistence of individual strains of Escherichia coli in the intestinal tract of man. J. Bact. 1952, 63, 47.

16. Miles, A. A. The Meaning of Pathogenicity in Mechanism of Microbial Pathogenicity. New York, Cambridge University Press, 1955, pp. 1-16. 